International Mathematical Forum, Vol. 8, 2013, no. 6, 283 - 288

\title{
A Semilinear Elliptic Problem with Neumann Condition on the Boundary
}

\author{
A. M. Marin \\ Faculty of Exact and Natural Sciences, University of Cartagena \\ Sede Piedra de Bolivar, Avenue of Consulado \\ Cartagena de Indias, Bolivar, Colombia

\section{R. D. Ortiz} \\ Faculty of Exact and Natural Sciences, University of Cartagena \\ Sede Piedra de Bolivar, Avenue of Consulado \\ Cartagena de Indias, Bolivar, Colombia

\section{J. A. Rodriguez} \\ Morelia Institute of Technology, Morelia, Mexico

\begin{abstract}
In this paper we study symmetric solutions for a semilinear elliptic problem with Neumann boundary condition to elliptic operators more general than the Laplacian on the unit ball in the n-dimensional Euclidean space with $n \geq 3$.
\end{abstract}

\section{Mathematics Subject Classification: 35J60}

Keywords: Nonlinear boundary value problems; Elliptic partial differential equations; Maximum principle; Symmetry; Uniqueness

\section{Introduction}

An interesting problem, from the point of view of the differential geometry and the elliptic differential equations, is the study of the behavior of solutions of

$$
\begin{aligned}
\Delta u & =f(u) \text { in } \mathrm{B}, \\
\frac{\partial u}{\partial \vec{n}} & =g(u) \text { on } \partial B
\end{aligned}
$$


where $B$ is the unitary ball in $\mathbb{R}^{n}, \frac{\partial}{\partial \vec{n}}$, denotes the outer normal derivative to $\partial B$, and $f, g$ are functions defined in $\mathbb{R}$. For $n \geq 3$ the problem (1) appears in the study of conformal deformations of the standard metric over the unitary ball $\bar{B}$ that have constant scalar curvature in $B$ and mean curvature on $\partial B$. In fact, if $G$ is a conformal metric to the standard metric $\delta_{i j}$ in $\bar{B}$, say $G=$ $u^{4 / n-2} \delta_{i j}$ with $u>0$ and smooth over $\bar{B}$, with constant scalar curvature $R_{G}$ in $B$ and constant mean curvature $h_{G}$ on $\partial B$, then $u$ satisfies the elliptic semilinear problem with Neumann condition,

$$
\begin{aligned}
\Delta u & =-\left(\frac{n-2}{4(n-1)}\right) R_{G} u^{\frac{n+2}{n-2}} \quad \text { in } \mathrm{B} \\
\frac{\partial u}{\partial \vec{n}} & =\frac{n-2}{2}\left\{h_{G} u^{\frac{n}{n-2}}-u\right\} \quad \text { on } \partial B .
\end{aligned}
$$

The professor José F. Escobar completely studied the set of such metrics. Furthermore, he proved that solutions are symmetric with respect to some point. (See [5], Theorem 2.1). Also in [6] there are studies with conformal metrics on the ball with zero scalar curvature and prescribed mean curvature on the boundary.

The maximum principle is one of the most used tools in the study of some differential equations of elliptic type. It is a generalization of the following well known theorem of the elemental calculus "If $f$ is a function of class $C^{2}$ in $[a, b]$ such that the second derivative is positive on $(a, b)$, then the maximum value of $f$ attains at the ends of $[a, b]$ ". It is important to point out that the maximum principle gives information about the global behavior of a function over a domain from the information of qualitative character in the boundary and without explicit knowledge of the same function. The maximum principle allows us, for example, to obtain uniqueness of solution of certain problems with conditions of Dirichlet and Neumann type. Also it allows to obtain a priori estimates for solutions. These reasons make interesting the study of the maximum principle on several forms and its generalizations and the Hopf lemma. For example a geometric version of the maximum principle allows us to compare locally surfaces that coincide at a point. On the other hand, the maximum principle and the Alexandrov reflection principle have been used to prove symmetries with respect to some point, some plane, symmetries of domain and to determine asymptotic-symmetric behavior of solutions of some elliptic problems. (See (Serrin [14], 1971); (Gidas, Ni and Nirenberg [7], 1979); (Gidas, Ni and Nirenberg [8], 1981); (Caffarelli, Gidas and Spruck [3], 1989); (Berestycki and Nirenberg [2], 1991)). The first person in use this technic was J. Serrin (See [14]). Serrin proved that: "If $u$ is a positive solution of the problem

$$
\Delta u=-1 \quad \text { en } \quad \Omega
$$


which

$$
u=0, \frac{\partial u}{\partial \nu}=\text { constant on } \partial \Omega,
$$

then $\Omega$ is a ball and $u$ is radially symmetric with respect to the center of $\Omega$ ". Using the ideas of Serrin and a version of the maximum principle for functions that do not change of sign, Gidas Ni and Nirenberg in [7] proved that: "If $\Omega$ is a ball, $f \in C^{1}(\mathbb{R})$ and $u$ is a positive solution of the problem,

$$
\Delta u+f(u)=0 \quad \text { on } \quad \Omega
$$

which is zero on the boundary, then $u$ is radially symmetric with respect to the center of the ball". Using the method of reflection and a version of maximum principle for thin domains Beresticky and Nirenberg in [2] made a generalization of paper [7]. Castro, Maya and Shivaji in [4] summarized the recent developments of semipositone problems.

We prove here that solutions of the elliptic problem with Neumann condition

$$
\begin{aligned}
a \Delta u+b \sum_{i=1}^{n} u_{i} & =f(u) \quad \text { in } \mathrm{B} \\
\frac{\partial u}{\partial \vec{n}} & =g(u) \text { on } \partial B
\end{aligned}
$$

are radially symmetric with respect to the origin, supposing nice behavior of $a, b, f$ and $g$ (See Theorem 2.1). Our proof shows that used technique in [2] , [7], [14] for the study of the symmetric solutions of the elliptic problem with Dirichlet condition

$$
\begin{aligned}
\Delta u+f(u) & =0 \text { in } \mathrm{B}, \\
u & =\varphi \text { on } \partial B, \\
u & >0 \text { on } B
\end{aligned}
$$

where $\bar{B}$ is the closed ball, can be applied in elliptic problems with Neumann condition.

\section{Main Result}

Now we announce the main theorem (see [9],[13]).

Theorem 2.1. Let $u \in C^{2}(B) \cap C^{0}(\bar{B})$ be a positive solution of the elliptic problem with Neumann condition

$$
\begin{aligned}
a \Delta u+b \sum_{i=1}^{n} u_{i} & =f(u) \quad \text { in } B, \\
\frac{\partial u}{\partial \vec{\eta}} & =g(u) \text { on } \partial B
\end{aligned}
$$


where $a: \bar{B} \rightarrow \mathbb{R}$ is a bounded function and symmetric with respect to the origin such that $a(x)>0$ for all $x \in \bar{B}$ and $b: \bar{B} \rightarrow \mathbb{R}$ is a bounded and odd function. Let $\vec{\eta}$ denote the normal vector to $\partial B$, and $f, g \in C(\mathbb{R})$. Suppose that

(a) $f$ is strictly increasing and

(b) $g$ is strictly decreasing.

Then, $u$ is radially symmetric with respect to the origin.

Proof. Let $\nu(x)=u\left(x^{\prime}\right), x \in \bar{B}$ where $x^{\prime}$ denotes the reflection of $x$ with respect to the hyperplane $x_{n}=0$. Then

$$
\begin{aligned}
a \Delta \nu+b \sum_{i=1}^{n} \nu_{i} & =f(\nu) \quad \text { in } B \\
\frac{\partial \nu}{\partial \vec{\eta}} & =g(\nu) \text { on } \partial B .
\end{aligned}
$$

Let $w$ be the function defined by $w(x)=u(x)-\nu(x) . x \in \bar{B}$. In consequence $w$ satisfies

$$
\begin{aligned}
a \Delta w+b \sum_{i=1}^{n} w_{i} & =f(u)-f(\nu) \text { in } B, \\
\frac{\partial w}{\partial \vec{\eta}} & =g(u)-g(\nu) \text { on } \partial B \\
w & =0 \text { in } \bar{B} \cap\left\{x_{n}=0\right\} .
\end{aligned}
$$

Since $w$ is continuous in $\bar{B}$, there exist points $x_{m}, x_{M} \in \bar{B}$ such that:

$$
w\left(x_{m}\right)=\min _{\bar{B}} w \quad \text { and } \quad w\left(x_{M}\right)=\max _{\bar{B}} w .
$$

See $x_{m}, x_{M}$ can not be in $\partial B$. Suppose $x_{M} \in \partial B$ and satisfies $w\left(x_{M}\right)>w(x)$ for all $x \in B$. Then, $\frac{\partial}{\partial \vec{\eta}} w\left(x_{M}\right) \geq 0$ and $w\left(x_{M}\right)>0$, in consequence

$$
g\left(u\left(x_{M}\right)\right)-g\left(\nu\left(x_{M}\right)\right) \geq 0 .
$$

Since $g$ is strictly decreasing we have $u\left(x_{M}\right) \leq \nu\left(x_{M}\right)$. Then, $w\left(x_{M}\right) \leq 0$ which contradicts $w\left(x_{M}\right)>0$.

Suppose $x_{m} \in \partial B$ and satisfies $w\left(x_{m}\right)<w(x)$ for all $x \in B$. Then, $\frac{\partial}{\partial \vec{\eta}} w\left(x_{m}\right) \leq 0$ and $w\left(x_{m}\right)<0$, in consequence

$$
g\left(u\left(x_{m}\right)\right)-g\left(\nu\left(x_{m}\right)\right) \leq 0 .
$$


Since $g$ is strictly decreasing we have $u\left(x_{m}\right) \geq \nu\left(x_{m}\right)$. Then, $w\left(x_{m}\right) \geq 0$ which contradicts $w\left(x_{m}\right)<0$.

Then $x_{m}$ can not be on $\partial B$. Hence $x_{m}, x_{M} \in B$. So

$$
\begin{gathered}
a\left(x_{m}\right) \Delta w\left(x_{m}\right)+b\left(x_{m}\right) \sum_{i=1}^{n} w_{i}\left(x_{m}\right) \geq 0 \quad \text { and } \\
a\left(x_{M}\right) \Delta w\left(x_{M}\right)+b\left(x_{M}\right) \sum_{i=1}^{n} w_{i}\left(x_{M}\right) \leq 0 .
\end{gathered}
$$

Since $f$ is strictly increasing

$$
u\left(x_{m}\right) \geq \nu\left(x_{m}\right) \quad \text { and } \quad u\left(x_{M}\right) \leq \nu\left(x_{M}\right)
$$

Then $w\left(x_{m}\right)=w\left(x_{M}\right)=0$. Hence $w \equiv 0$ in $\bar{B}$ and so $u$ is symmetric with respect to $x_{n}=0$. Since $B$ is the unit ball and we can apply the same arguments in all directions, it follows that $u$ is radially symmetric with respect to the origin.

ACKNOWLEDGEMENTS. The authors express their deep gratitude to CONACYT-México, Programa de Mejoramiento del Profesorado (PROMEP)México and Universidad de Cartagena for financial support.

\section{References}

[1] A. Alexandrov, Uniqueness theorems for surfaces in the large, V. Vestnik Leningrad. Uni. , 13 (1958), 5-8.

[2] H. Berestycki and L. Nirenberg, On the method of moving planes and the sliding method, Bol. Soc. Brasil. Mat., 221 (1991), 1-37.

[3] L. Cafarelli, B. Gidas and J. Spruck, Asymptotic symetry and local behavior of semilinear elliptic with critical sobolev growth, Comm. Pure. Math., 423 (1989), 271-297.

[4] A. Castro, C. Maya and R. Shivaji, Nonlinear eigenvalue problems with semipositone structure, Nonlinear Differential Equations, Electron. J. Diff. Eqns., Conf. 05, (2000) 33-49.

[5] J. Escobar, Uniquennes theorems on conformal deformations of metrics, Sobolev Inequalities, and a Eigenvalue Estimate. Comm. Pure. Math., 43 7 (1990), 857-883.

[6] J. Escobar and G. Garcia, Conformal metrics on the ball with zero scalar curvature and prescribed mean curvature on the boundary, Sobolev Inequalities, and a Eigenvalue Estimate. J. Func. Anal., 211 (2004), 71-152. 
[7] B. Gidas, Wei-Ming Ni and L. Nirenberg, Symetry and related properties via maximun principle, Comm Math. Phys., 68 (1979), 209-243.

[8] B. Gidas, Wei-Ming Ni and L. Nirenberg, Symetry of positive solutions of nonlinear elliptic equations in $\mathbb{R}^{N}$, in Mathematical Analysis and Applications, Part A, in: Adv. Math. Suppl. Stud. vol 7A, Academic Press, New York, (1981), 369-402.

[9] G. Garcia and J. Quintero, Simetria y un estudio cualitativo de soluciones de algunos problemas elípticos con condición de Neumann, Matemáticas: Enseñanza Universitaria, 31 (1993), 5-18.

[10] D. Gilbarg and N. Trudinger, Elliptic Partial Differential Equations of Second Order, Springer Verlag, Berlin, Heidelberg, New York, 1977.

[11] F. John, Partial Differential Equations, Springer Verlag, New York, 1982.

[12] M. Protter and H. Weinberger, Maximun Principle in Differential Equations, Springer-Verlag, New York, 1984.

[13] J. Quintero, Soluciones simetricas de algunos problemas elipticos, Revista Colombiana de Matemáticas., 27 (1993).

[14] J. Serrin, A Symetry problem in potential theory, Arch. Ration. Mech. Anal, 43 (1971), 304-318.

\section{Received: October, 2012}

